\title{
Sliding Free Lagrangian-Eulerian Finite Element Method
}

\author{
Junbo Cheng, Guiping Zhao, Zupeng Jia, Yibing Chen, Junxia Cheng, \\ Shuanghu Wang, and Wanzhi Wen \\ Institute of applied physics and computational mathematics, Beijing 100088, China \\ cheng-junbo@iapcm.ac.cn, magpzhao@yahoo.com.cn, zpjia@iapcm.ac.cn, \\ chen_yibing@iapcm.ac.cn, Cheng_Junxia@iapcm.ac.cn, \\ Wang_Shuanghu@iapcm.ac.cn, wen_wanzhi@iapcm.ac.cn
}

\begin{abstract}
People usually use arbitrary Lagrangian-Eulerian method to simulate the multi-phase flowing problems, but some numerical errors may be introduced during remapping. In this paper, sliding free Lagrangian-Eulerian finite element method(SFLEFEM) is developed. In SFLEFEM compressible Eulerian equations for moving mesh are dircretized without Lagrangian step and numerical experiments prove that SFLEFEM is convergent and stable.
\end{abstract}

\section{Description}

In Lagrangian approach, the mesh is embedded in the fluid and moves with it, So the precise material interface can be afforded. But when dealing with complex fluid, the mesh may be distorted seriously, the computation errors will increase quickly so that the computation is stopped. For solving the problem, people usually use rezoning and remapping techniques, but some errors may be introduced. So people can't use the rezoning and remapping techniques frequently.

In Eulerian approach, the mesh of grid points is fixed and so it can be used to calculate the problem of large deformation. Because the mesh is fixed, many other techniques, such as VOF[1], Front tracking [2], Level set [3] and Phase field [4], are used to track the moving material interface. But the precision of their capturing interface is lower than that of Lagrangian approach.

Because of these shortcomings, we introduce Sliding Free Lagrangian Eulerian Finite Element Method (SFLEFEM). Our objective is to deal with multimaterial physics problems with large-deformation and sliding interface. Numerical results show that SFLEFEM can simulate the multi-material problems with large-deformation and also can capture the material interface precisely.

SFLEFEM is different from Arbitrary Lagrangian-Eulerian(ALE) method. In ALE method, a Lagrangian step is first performed and the mesh is deformed according to the fluid flow. Then the improved mesh is generated based on the Lagrangian mesh and the solution is transferred from the Lagrangian mesh to the improved mesh. In SFLEFEM, a Lagrangian step is never performed. We directly calculate the fluid field from the time $t^{n}$ to the time $t^{n+1}$. Moreover, the 
grid points on the material interface or the boundary must move at the speed of Lagrangian velocity in the normal direction and can slide freely in the tangential direction. The tangential movement of boundary points can help us to generate the mesh of high quality.

In SFLEFEM, every material is calculated separately and the interaction between two neighboring materials is calculated by a new contact algorithm which is based on TENSOR contact algorithm. We will describe it in the future.

The equations of SFLEFEM are unsteady compressible Eulerian equations for moving grid points:

$$
\begin{array}{r}
\frac{\partial \rho}{\partial t}+\nabla \cdot(\rho \vec{D})=-\nabla \cdot(\rho \vec{w}), \\
\frac{\partial \rho e}{\partial t}+\nabla \cdot(\rho e \vec{D})=-p \nabla \cdot(\vec{v})-\nabla \cdot(\rho e \vec{w}), \\
\rho\left(\frac{\partial v_{z}}{\partial t}+\vec{D} \cdot \nabla v_{z}\right)=-\frac{\partial p}{\partial z}-\rho \vec{w} \cdot \nabla v_{z}, \\
\rho\left(\frac{\partial v_{r}}{\partial t}+\vec{D} \cdot \nabla v_{z}\right)=-\frac{\partial p}{\partial r}-\rho \vec{w} \cdot \nabla v_{r} .
\end{array}
$$

where $\vec{D}$ is the mesh velocity, $\vec{D}=\frac{d \vec{r}}{d t}, \vec{w}=\vec{v}-\vec{D}$.

Mass equation(1) is integrated on $\Omega(t)$ based upon Finite Volume method:

$$
\frac{M^{n+1}-M^{n}}{\triangle t}=\sum_{i=1}^{4} \rho_{i, i+1} \alpha_{i, i+1}\left(w_{r} \triangle z-w_{z} \triangle r\right)_{i, i+1},
$$

where $\alpha_{i, i+1}=\frac{\alpha_{i}+\alpha_{i+1}}{2}(\alpha=1$ for the planar problem and $\alpha=r$ for the axis symmetrical problem), $\left(w_{r}\right)_{i, i+1}=\frac{\left(w_{r}\right)_{i}+\left(w_{r}\right)_{i+1}}{2},\left(w_{z}\right)_{i, i+1}=\frac{\left(w_{z}\right)_{i}+\left(w_{z}\right)_{i+1}}{2}$, $\rho_{i, i+1}$ is the density along the boundary segment $(i, i+1)$. We use the secondorder MUSCL [5] scheme to calculate it.

Energy equation (2) is integrated on an one arbitrary mesh $\Omega(t)$ based upon the finite volume method and the Von Neumann and Richtmyer [6] viscosity $q^{n}$ is added for preventing from producing numerical oscillation, which yields

$$
\frac{E^{n+1}-E^{n}}{\Delta t}=-\left(\frac{p^{n}+p^{n+1}}{2}+q^{n}\right) \frac{\Delta V_{L}^{n+1}}{\Delta t}+g_{e}(t) .
$$

Where $\left.E=\int_{\Omega(t)} \rho e d V=e(t) M(t), \triangle V_{L}^{n+1}=V\left(\vec{r}^{n}+\Delta t \vec{v}^{n}\right)-V\left(\vec{r}^{n}\right)\right), g_{e}(t)=$ $-\int_{\Omega(t)} \nabla \cdot(\rho e \vec{w}) d V$. The discretization method for $g_{e}(t)$ is same as that for $-\int_{\Omega(t)} \nabla \cdot(\rho \vec{w}) d V$.

Momentum Equation(3) is discretized using finite element method, which gives

$$
\begin{aligned}
& \frac{v_{z}^{n+1}-v_{z}^{n}}{\Delta t} \sum_{i=1}^{4} \frac{1}{4} M_{i, A}^{n+\frac{1}{2}}=\sum_{i=1}^{4}\left(p_{i}^{n+\frac{1}{2}}+q_{i}^{n+\frac{1}{2}}\right) \frac{r_{i, 2}^{n+\frac{1}{2}}-r_{i, 4}^{n+\frac{1}{2}}}{2} \\
& -\sum_{i=1}^{4} \frac{\rho_{i}^{n+\frac{1}{2}}}{2}\left(v_{z, N}^{n}-\bar{v}_{z, i}^{n}\right)\left[\left(\bar{w}_{r}\right)_{i}\left(z_{i, 2}^{n+\frac{1}{2}}-z_{i, 4}^{n+\frac{1}{2}}\right)-\left(\bar{w}_{z}\right)_{i}\left(r_{i, 2}^{n+\frac{1}{2}}-r_{i, 4}^{n+\frac{1}{2}}\right)\right] .
\end{aligned}
$$


where $\left(\bar{w}_{r}\right)_{i}=\frac{1}{4} \sum_{k=1}^{4}\left(w_{r}\right)_{i, k},\left(\bar{w}_{z}\right)_{i}=\frac{1}{4} \sum_{k=1}^{4}\left(w_{z}\right)_{i, k},\left(\bar{v}_{z}\right)_{i}=\frac{1}{4} \sum_{k=1}^{4}\left(v_{z}\right)_{i, k}$. Now the discretization of equation (3) is finished. The methods of discretization for the equation (4) are same as that for the equation (3).

\section{Examples}

Saltzman piston problem [7] is used to test the ability of code to simulate the shock waves that are oblique to the mesh. Exact results for the problem are that the third shock arrives at the $\mathrm{z}$ coordinate of 0.95 and densities in front of and behind the third shock are 10 and 20 respectively. The pure Lagrangian methods(PLM) and SFLEFEM are used to simulate the problem and the results obtained from these two methods at time $\mathrm{t}=0.925$ are showed in Fig. 1.

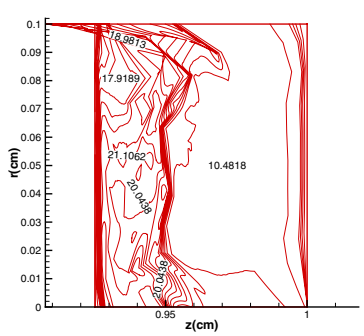

(a)

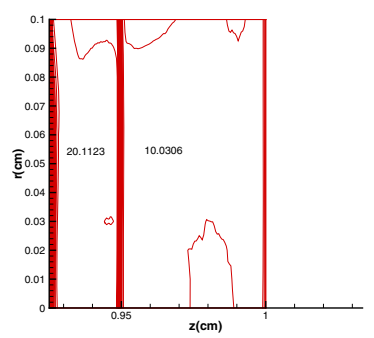

(b)

Fig. 1. (a)Density contours obtained from pure Lagrangian methods, (b)Density contours obtained from SFLEFEM

The figure of one-dimensional shock obtained from PLM is distorted seriously, especially along the top and bottom boundaries. But the density contours obtained from SFLEFEM give the right position of the shock and almost completely planar shock and the smaller density errors.

Dukowicz problem [7] is a shock refraction problem on an inclined interface and its sketch map is showed in Fig. 2. A piston moves from left to right with the constant velocity of 1.48 . On the right of the piston there are two ideal gases, the interface between two gases is aligned at $30^{\circ}$ to the horizontal.

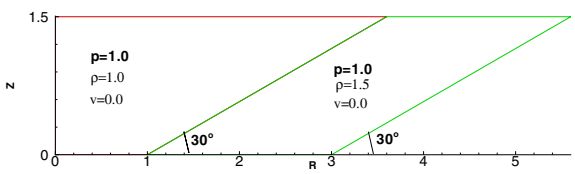

Fig. 2. Sketch map for Dukowicz problem 
The initial mesh is composed of two adjacent regions. The left region is a $36 \times 30$ mesh and the right region a $40 \times 30$ uniform mesh slanted at $60^{\circ}$. The density contours obtained by PLM and SFLEFEM are showed in Fig. 3 and Fig. 4 respectively at time $\mathrm{t}=1.3$. They are almost same as the density contours in 7]. The main difference is where the interface arrives at on the lower boundary.

We also use the refiner mesh $(72 \times 60$ zones in the left region and $80 \times 60$ zones in the right region) to calculate the problem. The interface location on the lower boundary at time $t=1.3$ are showed in table 1 . Table 1 shows the interface location from SFLEFEM is same even if using different mesh. That shows SFLEFEM is convergent.

Table 1. Interface location on lower boundary

\begin{tabular}{|c|c|c|c|c|}
\hline $\begin{array}{c}\text { Algorithm } \\
\text { mesh }\end{array}$ & $\begin{array}{c}\text { PLM } \\
\text { coarse mesh }\end{array}$ & $\begin{array}{c}\text { SFLEFEM } \\
\text { coarse mesh }\end{array}$ & $\begin{array}{c}\text { PLM } \\
\text { refine mesh }\end{array}$ & $\begin{array}{c}\text { SFLEFEM } \\
\text { refine mesh }\end{array}$ \\
\hline Interface position on lower boundary & 1.99 & 2.05 & 2.04 & 2.05 \\
\hline
\end{tabular}

Noh problem [7] is used to test the ability of the code to keep symmetrization. In Noh problem, an ideal gas moves in an initial unit inward radial velocity. Exact solution and results obtained from PLM and SFLEFEM with a polar mesh of $50 \times$ 50 are showed in Fig. 5. Fig. 5 shows two methods give the right shock location and they can keep the circular shock the almost complete symmetrization, but the density errors of SFLEFEM are smaller.

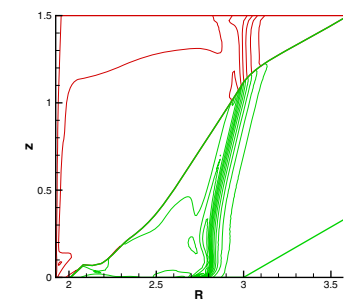

Fig. 3. Density contours from PLM

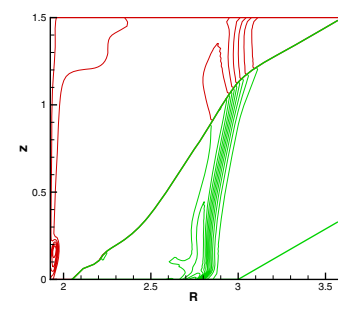

Fig. 4. Density from SFLEFEM

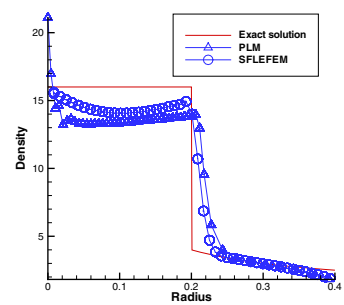

contours Fig. 5. Density distribution versus the radius of the center of all cells

\section{Summary and Discussion}

SFLEFEM has both the character of Lagrangian approach and the attribute of Euler approach. In SFLEFEM grid points can move free, which does not introduce new numerical errors. Capturing the shock waves and the interface precisely and decreasing the density errors prove the efficiency of SFLEFEM. But the more development of SFLEFEM is necessary, especially for the time discretization of momentum equations. We will use high order R-K methods to discretize the time derivatives in the momentum equations in future. 


\section{References}

1. Hirt C. W. and Nichols B. D.: Volume of fluid(VOF) method for the dynamics of free boundary, J. Comput. Phys. 39 (1981) 201-225.

2. Chen I. L. and Glimm J.: Front tracking for gas dynamics, J. Comput. Phys. 62 (1986) 83-110.

3. Osher S. and Sethian J. A., Fronts propagating with curvature depend speed: Algorithm based on Hamilton-Jacobi formulation, J. Comput. Phys. 79 (1988) 12

4. Antannovskii L. K., A phase field model of capillavity.

5. David J. Benson.: An efficient, accurate, simple ALE method for nonlinear finite element programs, Comm. Pure Appl. Math. 72 (1989) 305-350

6. Von Neumann and R. D. Richtmyer.: A method for the numerical calculation of hydrodynamics shocks, J. Appl. Phys. 21(1950)

7. J. Campbell and M. Shashkov.: A Tensor Artificial Viscosity using a Mimetic Finite Difference Algorithm, Los Alamos NM 87545, April 2000, LA-UR-00-2900 